\title{
A game-based neurofeedback training system to enhance cognitive performance in healthy elderly subjects and in patients with amnestic mild cognitive impairment
}

This article was published in the following Dove Medical Press journal:

Clinical Interventions in Aging

\author{
Suwicha Jirayucharoensak ${ }^{1,2}$ \\ Pasin Israsena' \\ Setha Pan-ngum ${ }^{2}$ \\ Solaphat Hemrungrojn ${ }^{3}$ \\ Michael Maes ${ }^{3}$ \\ 'Neural Signal Processing Research \\ Team, Artificial Intelligence Research \\ Unit, National Electronics and \\ Computer Technology Center, Pathum \\ Thani I2 I20, Thailand; ${ }^{2}$ Department \\ of Computer Engineering, Faculty of \\ Engineering, Chulalongkorn University, \\ Bangkok 10330, Thailand; ${ }^{3}$ Department \\ of Psychiatry, Faculty of Medicine, \\ Chulalongkorn University, Bangkok \\ 10330, Thailand
}

Introduction: This study examines the clinical efficacy of a game-based neurofeedback training (NFT) system to enhance cognitive performance in patients with amnestic mild cognitive impairment (aMCI) and healthy elderly subjects. The NFT system includes five games designed to improve attention span and cognitive performance. The system estimates attention levels by investigating the power spectrum of Beta and Alpha bands.

Methods: We recruited 65 women with aMCI and 54 healthy elderly women. All participants were treated with care as usual (CAU); 58 were treated with CAU + NFT (20 sessions of 30 minutes each, 2-3 sessions per week), 36 with CAU + exergame-based training, while 25 patients had only CAU. Cognitive functions were assessed using the Cambridge Neuropsychological Test Automated Battery both before and after treatment.

Results: NFT significantly improved rapid visual processing and spatial working memory (SWM), including strategy, when compared with exergame training and no active treatment. aMCI was characterized by impairments in SWM (including strategy), pattern recognition memory, and delayed matching to samples.

Conclusion: In conclusion, treatment with NFT improves sustained attention and SWM. Nevertheless, NFT had no significant effect on pattern recognition memory and short-term visual memory, which are the other hallmarks of aMCI. The NFT system used here may selectively improve sustained attention, strategy, and executive functions, but not other cognitive impairments, which characterize aMCI in women.

Keywords: amnestic mild cognitive impairment, neurofeedback, cognition, executive functions, aging, serious gaming, brain-computer interface

\section{Introduction}

Brain-computer interface (BCI) provides a promising technology that allows humans to control their brain waves through an external device. ${ }^{1-4}$ BCI may be employed to improve neurocognitive functions, such as attention span and working memory. ${ }^{5-8}$ The neurofeedback training (NFT) system acquires brain wave activities from an electroencephalogram (EEG) amplifier and then extracts EEG features to define states of cognition. The system visualizes the estimated cognitive state of the subjects who are able to recognize their own current state of cognition. Consequently, participants may learn how to modulate their brain EEG activities to meet the goals of the training protocol in each training session.
Correspondence: Pasin Israsena National Electronics and Computer Technology Center, II 2 Thailand Science Park, Klong Luang, Pathum Thani I2I20, Thailand

Tel +6625646900 ext 247I

Fax +6625646902

Email pasin.israsena@nectec.or.th 
Knowledge of NFT systems has undergone considerable developments by studying how to improve the cognitive state and attention using NFT in, for example, children with Attention-Deficit Hyperactivity Disorder (ADHD). ${ }^{9-12}$ Some studies delineated the association between learning problems or sustained attention and cognitive performance in ADHD. ${ }^{13,14}$ Moreover, NFT systems were designed with the aim to improve cognitive performance not only in children with ADHD but also in elderly individuals. For example, Becerra et al examined the effect of NFT, which targeted theta reduction, on neurocognitive performance in healthy elderly people. ${ }^{15}$ They randomly assigned 14 subjects to either active NFT or sham treatment and found that subjects in the experimental group exhibited greater improvement in EEG and behavioral measurements. Zoefel et al used an NFT system that targets the amplitude of the upper alpha frequency band by playing mental rotation games. ${ }^{16}$ The authors found that 11 of 14 undergrad students who had attended five NFT sessions in 1 week significantly improved their cognitive performance when compared with non-NFT control group. Haddadi et al examined an NFT system to enhance learning and memory in patients with cognitive impairment. ${ }^{17}$ Their NFT protocol (40 sessions, 45 minutes/day, and 3 sessions a week) increased beta wave frequency and decreased theta wave frequency in association with improved scores on the Wechsler Intelligence Scale for Children and Child Behavior Checklist. Engelbregt et al evaluated short- and long-term effects of frontal beta EEG-NFT in healthy subjects and found that frontal beta activities increased after 15 sessions of 45-minutes NFT intervention and that these effects remained stable for at least 3 years. ${ }^{18}$ Gruzelier provided an intensive review of cognitive and affective outcomes of EEG-NFT in healthy subjects and showed that outcome gains included memory, executive functions, and sustained attention. ${ }^{19}$ There are also some studies that evaluated the effects of NFT in patients with Alzheimer's Disease (AD). Luijmes et al examined positive outcomes of theta-decreased NFT in patients with AD by performing 30 intervention sessions and found improved recall of information and recognition, ${ }^{20}$ although other cognitive functions did not change. Hohenfeld et al studied cognitive functions in association with brain activity and found that NFT improved visuospatial memory performance; however, no significant changes in brain activities were found..$^{21}$ Recently, it was shown that NFT may improve memory performance in association with peak alpha frequency in patients with mild cognitive impairment (MCI). ${ }^{22}$ Nevertheless, there are no data whether, in amnestic MCI (aMCI), NFT has an effect on other cognitive functions including spatial working memory
(SWM), strategy, visual pattern recognition and memory, sustained attention, and attention span.

Hence, the main aim of this study was to examine whether a newly developed game-based NFT system may enhance neurocognitive performance in healthy elderly subjects and patients with aMCI. To evaluate the effects of NFT, we examined the tests scores of key cognitive domains as measured with the Cambridge Neuropsychological Test Automated Battery (CANTAB) both before and after NFT and compared the results with exergame-based cognitive training and care as usual (CAU). We decided to use exergame as a control treatment because the latter is a popular treatment that may enhance cognition and may help some patients with MCI. ${ }^{23}$

\section{Methods}

\section{Participants}

In this study, we recruited 119 women, 65 women with aMCI, and 54 healthy elderly women. The aMCI participants consulted the Dementia Clinic, Department of Psychiatry, King Chulalongkorn Memorial Hospital, Chulalongkorn University, Bangkok, Thailand, for subjective memory complaints. The normal controls were recruited from people who worked as volunteers at the same department. The diagnosis of aMCI was made by a senior neuropsychiatrist specialized in aMCI and dementia (author HS) after taking clinical history, neurological and physical examinations, and administration of the Clinical Dementia Rating (CDR).${ }^{24} \mathrm{We}$ made the diagnosis of aMCI by employing Petersen criteria and recruited only those patients who were not diagnosed to have dementia by using the National Institute of Neurological and Communicative Disorders and Stroke and the Alzheimer's Disease and Related Disorder Association criteria (NINCDS-ADRDA). ${ }^{25,26}$ Moreover, only patients with a CDR score of 0.5 and a CDR memory component score of 0.5 were recruited, while the Mini-Mental State Examination (MMSE) score was not lower than the dementia cutoff score determined by stratification for education. All healthy elderly controls had a CDR score of 0 .

All subjects showed adequate verbal expression, visual and hearing abilities, and had at least 4 years of education. We included only those participants who were willing to participate in 20 NFT or exergame sessions. We excluded subjects with axis 1 diagnosis according to DSM-5 criteria such as dementia, schizophrenia, and psychoorganic syndromes. ${ }^{27}$ Other exclusion criteria were neurologic disorders, including brain tumors, stroke, multiple sclerosis, Parkinson's disease, and epilepsy; use of acetyl cholinesterase inhibitors (such as donepezil, galantamine, and rivastigmine); substance abuse 
disorders or substance dependence; and any axis 1 psychiatric diagnosis according to DSM-5. The study was approved by the Institutional Review Board, Faculty of Medicine, Chulalongkorn University, Bangkok, Thailand (no 593/57). All participants provided written informed consent, and the study was conducted in accordance with the Declaration of Helsinki.

\section{Measurements}

We employed the Thai Mental State Examination (TMSE) and the Montreal Cognitive Assessment (MOCA) to assess severity of aMCI. The TMSE is the MMSE validated for a Thai population and contains six basic subtests concerning orientation ( 6 points), registration ( 3 points), attention (5 points), calculation ( 3 points), language (10 points), and word recall ( 3 points); the total score ranges from 0 to 30 . The TMSE assesses global cognitive function and is useful in screening cognitive impairment. ${ }^{28}$ The MOCA distinguishes subjects with normal age-related cognitive decline from subjects with MCI. ${ }^{29}$ This test assesses several cognitive domains, including visuospatial (4 points), executive functions ( 4 points), language abilities ( 6 points), attention (5 points), memory (5 points), and orientation ( 6 points) and the total score is 30 . The MOCA puts more emphasis on frontal executive functioning and attention than the TMSE and can be used to detect neurocognitive dysfunctions in individuals who score within the normal range on the TMSE.

\section{Experimental protocol}

The NFT intervention group consisted of 58 subjects (32 with aMCI and 26 normal elderly controls); the exergame treatment group consisted of 36 subjects (19 with aMCI and 17 normal controls), while the CAU group consisted of 25 subjects (14 with aMCI and 11 normal elderly controls). Subjects were randomized so that the number of NFT subjects was the same as the number of exergame treated + no treated subjects. Subjects were additionally recruited to comprise around 55\% aMCI subjects and 45\% elderly controls. The experiment protocol is depicted in Figure 1. The baseline (pretreatment) neurocognitive functioning was measured using the CANTAB tests. Subsequently, subjects in both active intervention groups (CAU + NFT and CAU + Exergame) underwent 20 intervention sessions, 2-3 sessions a week, and then underwent a second cognitive evaluation using the CANTAB. Subjects in the control group had a second neurocognitive test after the same period of time (3 months).

We employed the CANTAB as a rapid, accurate, and language-independent assessment of neurocognitive

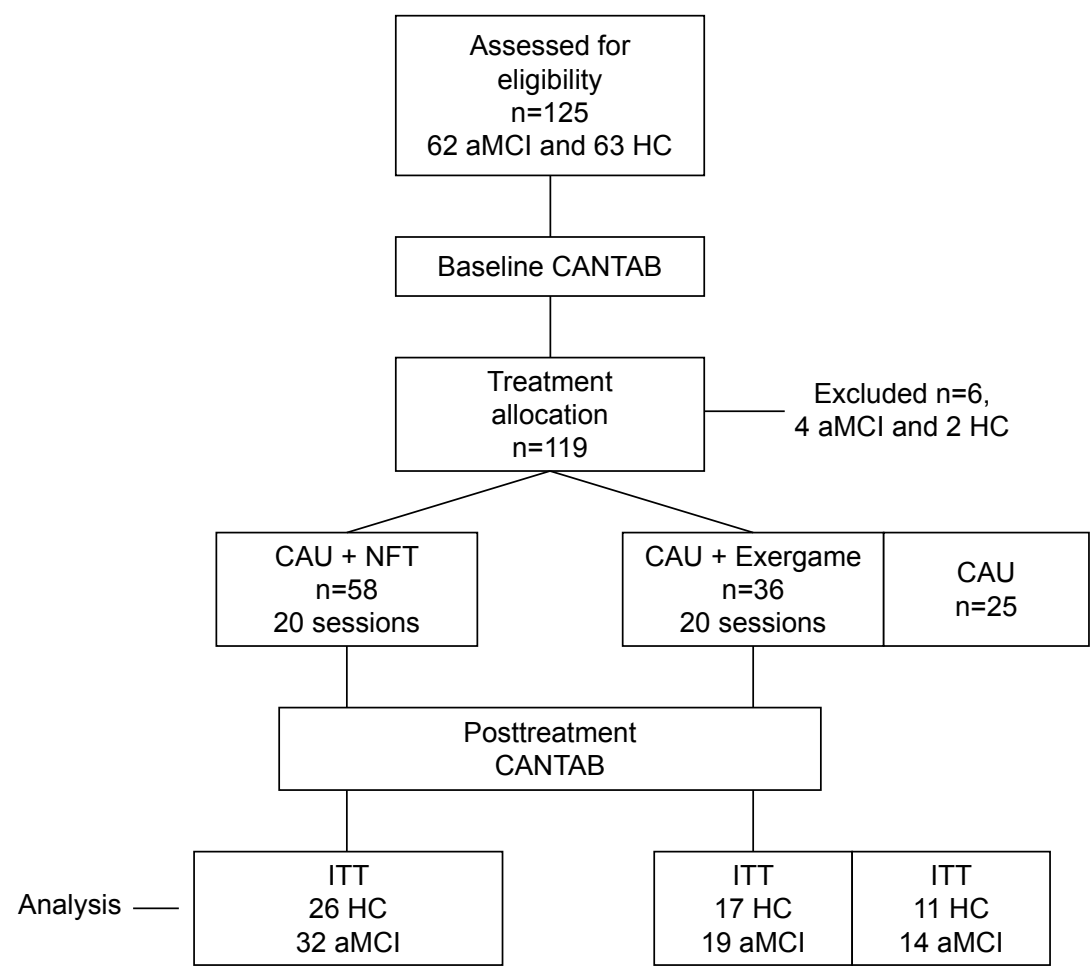

Figure I The experiment protocol, CONSORT flow diagram.

Abbreviations: aMCl, patients with amnestic mild cognitive impairment; CANTAB, Cambridge Neuropsychological Test Automated Battery; CAU, care as usual; HC, healthy controls; NFT, neurofeedback training; ITT, intention-to-treat analysis. 
functioning. ${ }^{30}$ We used various CANTAB tests denoted by CANTAB as key cognitive tests for aMCI, including SWM, Rapid Visual information Processing (RVP), Pattern Delayed Matching to Sample (DMS), Pattern Recognition Memory (PRM), and Spatial Span Length (SSP). SWM probes the ability to retain spatial information and manipulate remembered items in working memory by playing a self-ordered task. We used SWM Between Error (SWM_BER) and SWM Strategy (SWM_STR), which are probes that measure the efficient strategy for completing the task. PRM probes visual PRM. PRM Correct (PRM_COR) is the number of correct answers of the subject. DMS probes the ability to perform simultaneous and delayed matching to sample, and probes short term visual memory and visual recognition memory. A complex visual pattern is displayed on the screen and then, after a brief delay, the subject is required to select the matched pattern from four patterns. DMS_COR (Total Correct) is the total number of trials in which the subjects select the correct stimulus on their first response. DMS_MdCOR (Median Correct Latency) is the median latency in all trials where the subject selected the correct stimulus. DMS_PER (Percent Correct) is the percentage of occasions upon which the subject selected the correct stimulus in trials. RVP probes visual sustained attention capability. RVP_A' assesses how effective the subject is at detecting target sequences, while RVP_MdL (Median Latency) is a measure of the median time taken to respond. SSP probes the subject's ability to assess working memory and attention span. We used the SSP_SPAN (Span Length), namely the longest sequence successfully recalled by the subject.

\section{NFT-based cognitive training system}

The block diagram of the NFT system is depicted in Figure 2. The system acquires raw EEG signals from an Emotiv headset. ${ }^{31}$ Before the system estimates the cognitive state of attention, all EEG artifacts must be removed from the raw brain signals. The system exploits Independent Component Analysis (ICA), with Lifting Wavelet transform (LWT) collaboration to enhance the efficiency of the artifact removal, and the cognitive state of attention can then be estimated by measuring the relative power ratio of Beta/Alpha frequency bands. Subsequently, the system will adjust the character of objects on the screen corresponding to the estimated attention level. Therefore, subjects are able to recognize their own current state of attention and stay focused on the target object throughout each training session.

\section{EEG signal acquisition}

The Emotiv EEG headset was utilized for EEG signal acquisition. In this study, the system acquired raw EEG signal

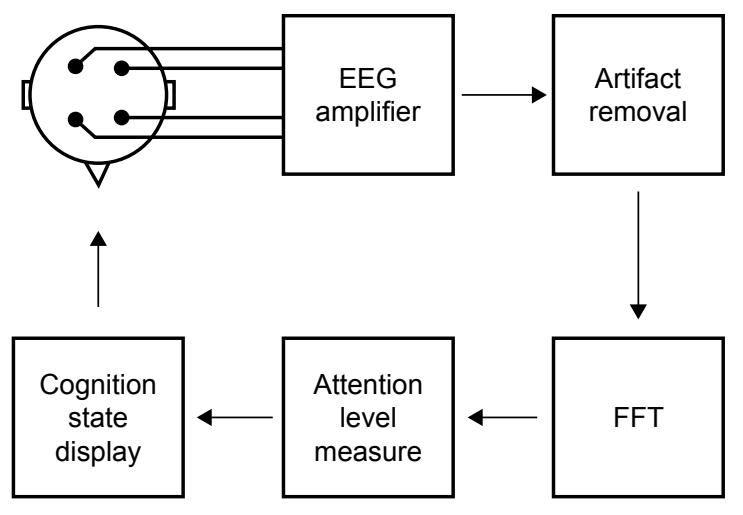

Figure 2 NFT system process flow.

Notes: NFT processing pipeline starts with a four-channel raw EEG signal acquisition, and then artifact removal system provides clean EEG signals before computing power spectrum for each band using FFT. The system examines brain activity changes to determine attention state. Then, subjects can recognize their own cognitive state of attention through games on the display.

Abbreviations: EEG, electroencephalogram; FFT, Fast Fourier Transform; NFT, neurofeedback training.

at $128 \mathrm{~Hz}$ sampling rate from two positions at frontal lobe region, $\mathrm{AF} 3$ and $\mathrm{AF} 4$, and other two positions at occipital lope region, $\mathrm{O} 1$ and $\mathrm{O} 2$. The electrodes were placed according to the international 10-20 system locations. The raw EEG signal was band-pass filtered in the frequency range of $0.1-45 \mathrm{~Hz}$.

In this study, all raw EEG signals from four channels were recorded during NFT intervention sessions for further analysis. We investigated the effects of the NFT system on targeted EEG frequency band, ie, Beta and Alpha bands. The power spectrum of five bands (Delta $1-4 \mathrm{~Hz}$, Theta 4-8 Hz, Alpha 8-12 Hz, Beta 12-32 Hz, and Gamma 32-45 Hz) was calculated by utilizing MATLAB Fast Fourier Transform function with a Hanning window, and the window size was 256 samples. A relative power of each band was compared with the summation of power spectrum of all five bands, as shown in Equation 1. In each NFT session, the system recorded EEG signals at $\mathrm{AF} 3, \mathrm{AF} 4, \mathrm{O} 1$, and $\mathrm{O} 2$ for calculating the relative power spectrum of Beta and Alpha frequency bands. The relative power ratio of Beta/Alpha was utilized as an indicator of the attention level.

$$
\text { Relative power }=\frac{\text { Power }(\text { band })}{\sum_{i=1}^{5} \operatorname{Power}(i)}
$$

\section{EEG artifact removal}

The attention level computation of the NFT system must be reliable to generate accurate neurofeedback responses in real time. All EEG artifacts must be removed or suppressed prior to attention state estimation. The EEG artifacts are caused mainly by electrooculography (EOG) and electromyography artifacts. These artifacts make the system's cognitive state 
estimation less reliable. Ocular artifacts (EOG) are caused by eye blinks and eye movements. Eye blink is one of the most eminent artifacts, which induces tremendously high amplitude signals compared to those of brain neural signals. Only a small amount of eye blinks can significantly affect the accuracy of EEG-based data classification. The characteristic of EOG dominates EEG signals in the $0-5 \mathrm{~Hz}$ frequency band. ${ }^{32}$ The muscular artifacts have wider frequency bands and dominate EEG signals in the 15-35 Hz frequency band. ${ }^{33,34}$ Therefore, we focus on two frequency bands, ie, $0-5$ and $15-35 \mathrm{~Hz}$, to identify ocular and muscular artifacts, respectively.

We used ICA with LWT as EEG artifact suppression technique. ${ }^{35}$ The technique employs LWT, instead of conventional wavelet transform, to elaborately extract neural signals from artifact components. The computation complexity of LWT is considerably lower than that of conventional wavelet transform. Consequently, the EEG artifact suppression technique has the capability to eliminate ocular and muscular artifacts from multichannel EEG brain signals in real time. It is critical for NFT systems to automatically suppress the EEG artifacts in real time because subjects must be able to recognize their own current cognitive state.

\section{Attention level estimation}

The NFT system measures raw EEG signals from the Emotiv EEG headset at four positions, ie, AF3, AF4, O1, and O2. The EEG amplifier has a sampling rate at $128 \mathrm{~Hz}$ and $0.1-45 \mathrm{~Hz}$ bandpass filter. Each processed data block size is assigned to 64 samples. However, the system implements a buffer of 192 previous samples to make the cognitive state estimation more accurate. Therefore, in every 0.5 seconds, the system runs the EEG artifact removal algorithm on these 256 samples and computes the value of relative power spectrum of the Beta and Alpha bands of each channel. Consequently, the average value of relative power spectrum of these two bands at four positions (AF3, AF4, O1, and $\mathrm{O} 2$ ) was calculated to measure the attention level.

A previous study of our research team investigating EEG electrode location to adequately assess cognitive state of attention found that AF3 was one of the best locations to recognize the attended state, while the AF4 channel was selected based on a simple symmetry design of a low-cost minimal channel EEG headset in the next project. ${ }^{36}$ Moreover, $\mathrm{O} 1$ and $\mathrm{O} 2$ locations were selected for ICA artifact removal purposes, because the raw EEG signals at $\mathrm{O} 1$ and $\mathrm{O} 2$ may be less affected by eye-blink artifacts.

The cognitive state of attention can be identified by investigating crucial EEG modifications of Beta and Alpha bands. During the state of attention, the power spectrum of the Alpha band is lower and that of the Beta band is higher than that of the rest state. ${ }^{37,38}$ The definition of attention level is shown in Equation 2.

$$
\text { Attention }=\frac{K_{\beta} * \mathrm{P}_{\beta}}{K_{\alpha} * \mathrm{P}_{\alpha}}
$$

where $K_{\beta}$ is the constant for scaling the value of Beta band, $P_{\beta}$ is the relative power spectrum of Beta band, $K_{\alpha}$ is the constant for scaling the value of Alpha band power, and $P_{\alpha}$ is the relative power spectrum of Alpha band.

The NFT system initially estimates upper and lower threshold levels by measuring the attention level of a subject during focused and relaxed states. During the focused state, subjects were asked to watch a particular object in the picture displayed on screen for 1 minute. During the relaxed state, the system displayed a picture of a beautiful and calm sea view with soothing music. Subsequently, the system uses these two levels as triggering threshold levels (upper and lower thresholds). If the value of attention is above the upper threshold level, the game will give a reward, ie, faster car speed. On the other hand, if the value is below the lower threshold level, the game will provide a penalty, ie, slower car speed.

Inter-subject and inter-session variations of EEG signals significantly affect accuracy and performance of BCI applications. To alleviate these variation effects, each subject was required to perform a short calibration session prior to playing NFT games in each intervention session. During individual calibration, the system calculates $K_{\beta}$ and $K_{\alpha}$ values by considering the power modification between attended and unattended states on Beta and Alpha bands. These constants are employed to make the modifications more sensitive to detect the cognitive state of attention. Subsequently, these constant values will be adjusted to obtain a proper scale (0-100) of attention level. Occasionally, the power spectrum of Beta band in the relaxed state has a greater value than that in the attended state. The power spectrum of Alpha band in the attended state has a greater value than that in the relaxed state. When one of these two situations occurs, the system needs to perform the individual calibration process again to acquire the proper threshold levels.

In addition, EEG signals are nonstationary and adversely affect the system's performance during NFT intervention in each session. To alleviate the nonstationary effect, the NFT system estimates the value of the attention levels by averaging the current attention value with its three previous values. In other words, the system calculates the moving 
average value of attention level in the window of 2 seconds (using four of consecutive attention values) and then uses this moving average value to adjust the displayed object in the games. Moreover, during playing the games, these triggering threshold levels were automatically adjusted according to the rate of success of the participant in order to avoid causing a sense of frustration or failure. If these thresholds were much higher than the current attention level, the program gradually adjusts these thresholds lower and vice versa.

\section{Cognitive state display}

The game-based NFT system estimates the subject's sustained attention levels by examining power spectrum of Beta and Alpha bands and then adjusts the character of the target object with the estimated attention level, ie, speed of car. This NFT mechanism allows subjects to recognize their current attention state and attempt to remain in the attended state throughout the training sessions. As such the subjects learn to modulate their brain EEG activity. All NFT intervention sessions were arranged in a particular room decorated for relaxing atmosphere as shown in Figure $3 \mathrm{~A}$. The subject sat on a comfortable chair in front of a 23-in. LED display with $80-100 \mathrm{~cm}$ distance. Prior to the first intervention session, the participants were required to watch a tutorial that explains how the NFT system works and how to play NFT games (see Figure 3B). Note that people who appear in the figures provided written informed consent for publication of their images.

In our study, both the healthy and aMCI subjects in the intervention groups underwent 20 NFT interventions, 2-3 sessions a week. In each session, subjects had to perform five 4-minute NFT interventions. Between each game, there was a 2-minute intermission for participant's comfort. The NFT system for attention consists of five games and the participants played all games during each session. Playing games clearly entertained the subjects and allowed them to train for longer periods of time.

There are five games in our NFT system for cognitive performance enhancement. The first NFT game called "Run Run", in Figure 4A, provides a proper NFT intervention by increasing bear's running speed when the player focuses on the game. On the other hand, if the player does not focus on the game, the speed of running will be gradually decreased. During running trial, the bear can jump to collect coins, if he runs fast enough. The second game is called "Sunshine Day", in Figure 4B. There is a girl in a garden. If the player focuses on her or cheers her up, the girl will be happy and jump in a good mood. Otherwise, the girl will be in a sad mood. The third game called "Cast Away", in Figure 4C, aims to adjust cooking fire according to the player's attention level. If the fire is strong, all food will be cooked fast, and so game scores will be high. The fourth game is called "Paper Plane", in Figure 4D. The speed of the plane will be adjusted along with the attention value of the player. If the attention value is high, the plane will be able to fly away from obstacles. In the fifth game called "Shoot Shoot", in Figure 4E, if the attention value is above an adaptive threshold level, the boy will shoot the basketball.

\section{Exergame-based cognitive training system}

The exergame was developed for older adults to enhance their cognitive performance by performing physical training
A

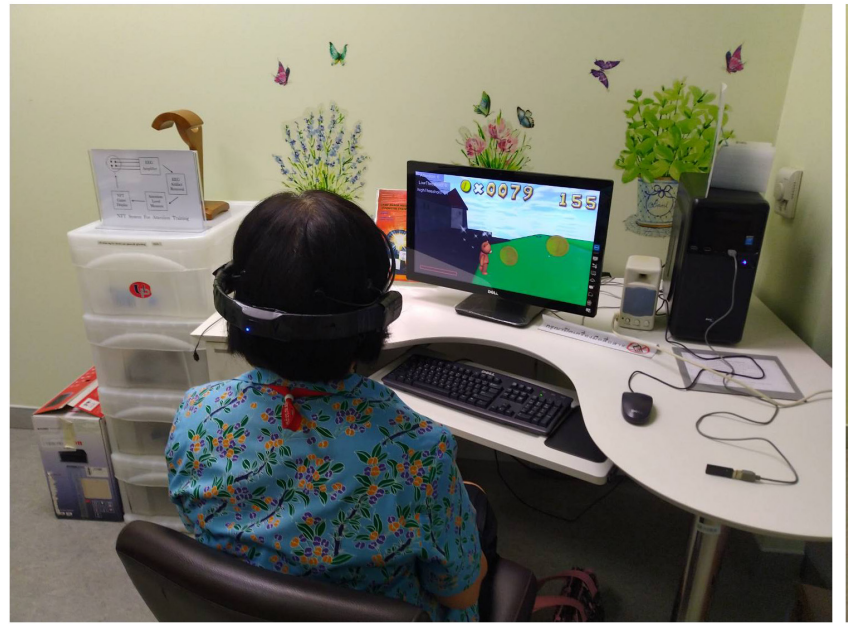

B

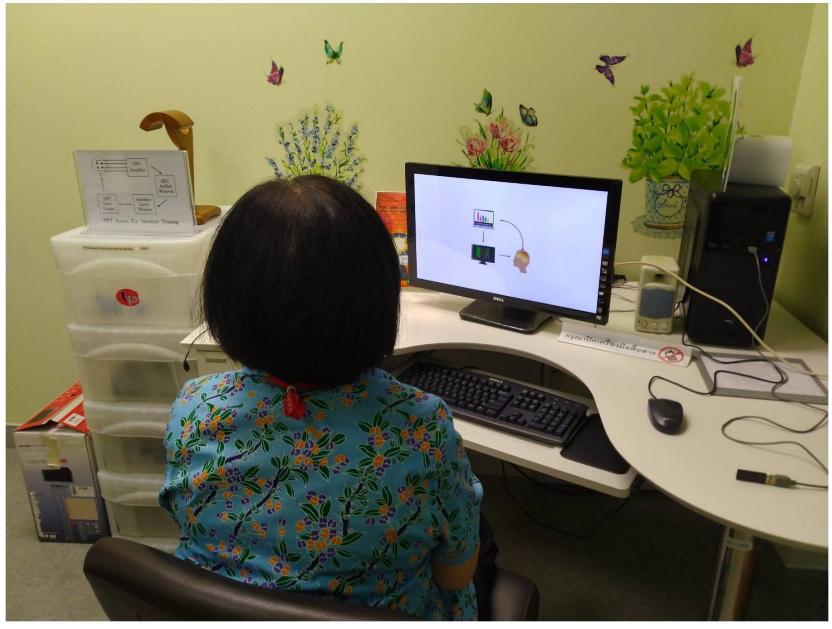

Figure 3 Neurofeedback intervention sessions.

Notes: (A) NFT intervention session. (B) NFT tutorial session.

Abbreviation: NFT, neurofeedback training. 


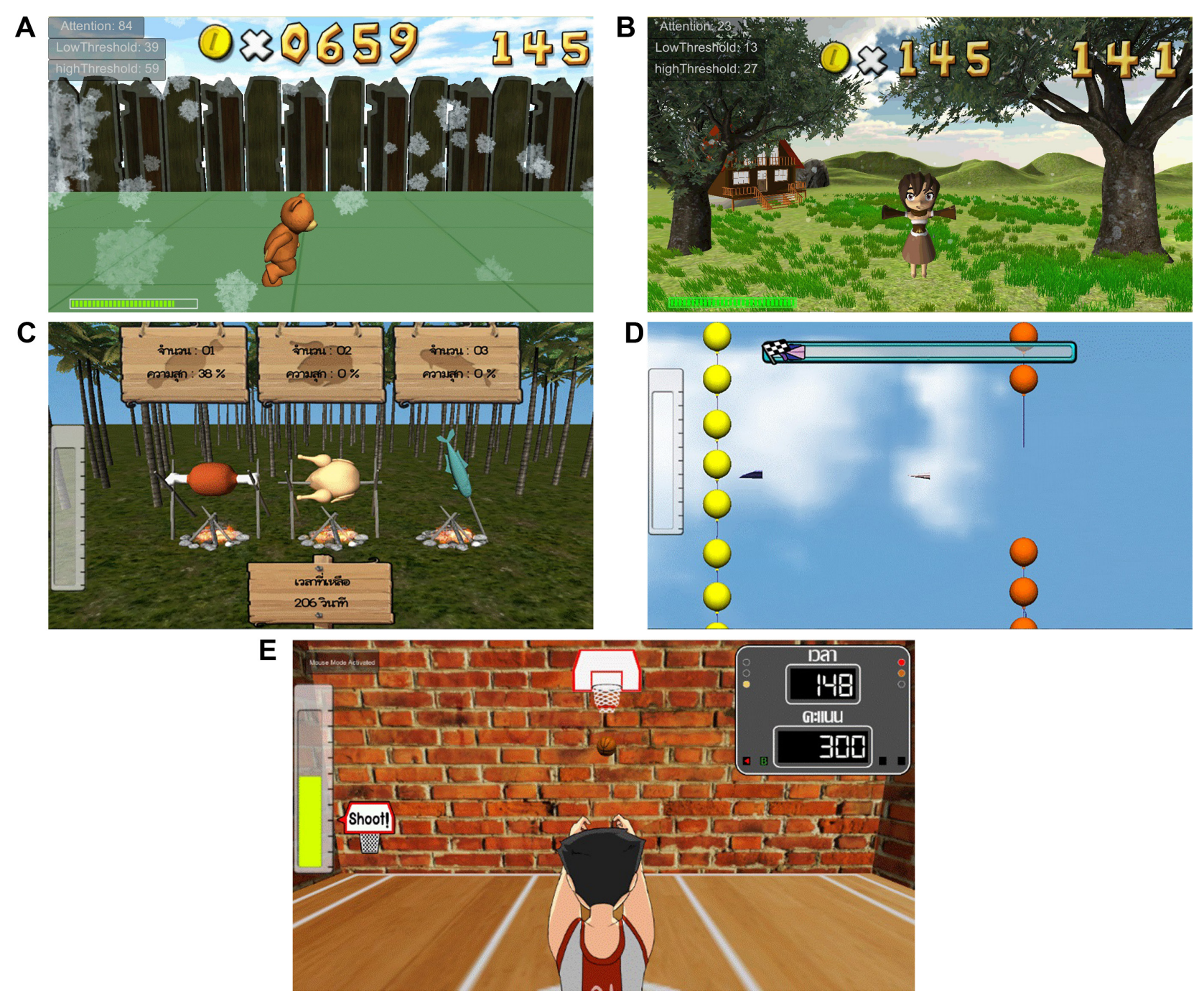

Figure 4 Neurofeedback games.

Note: (A) Run Run, (B) Sun Shine Day, (C) Cast Away, (D) Paper Plane, and (E) Shoot Shoot.

and cognitive training simultaneously. The system uses Microsoft Kinect v2 to detect physical movement for controlling games. There are five games depicted in Figure 5. The first game is called "Dogsperate", in Figure 5A. Players need to control dog's movement by moving their body to left or right direction to avoid obstacles. The players can control the dog to jump over obstacles by waiving their left or right hand. In the second game called "Penguin", in Figure 5B, the penguin's movement direction is controlled by leaning direction of player to pick fishes up along the trial in order to practice body balancing. In the third game (Figure 5C), players need to practice eye-hand coordination by moving their left and right hands to hit all points in sequence. To enhance episodic memory and long-term memory recall capacities, in the fourth game as shown in Figure 5D, players need to reorder the sequence of their own pictures. In the final game (Figure 5E), to enhance numerical cognition and reasoning skill, players perform mathematic operation by moving the correct balloon number to equation presented above.

All exergame-based cognitive training intervention sessions were arranged in an experiment room. The training room had a big screen displayed on the wall. The floor of the training room was fully installed with shockproof cushions to prevent any injuries during training sessions. Figure 6 shows some exergame intervention sessions during the experiment.

\section{Statistics}

Analysis of contingency tables (chi-squared test) was used to assess associations between categorical variables and ANOVA was used to assess differences in continuous variables between treatment groups. The primary outcome analysis is a repeated measurement generalized estimating equation (GEE) analysis with the repeated measurements of 


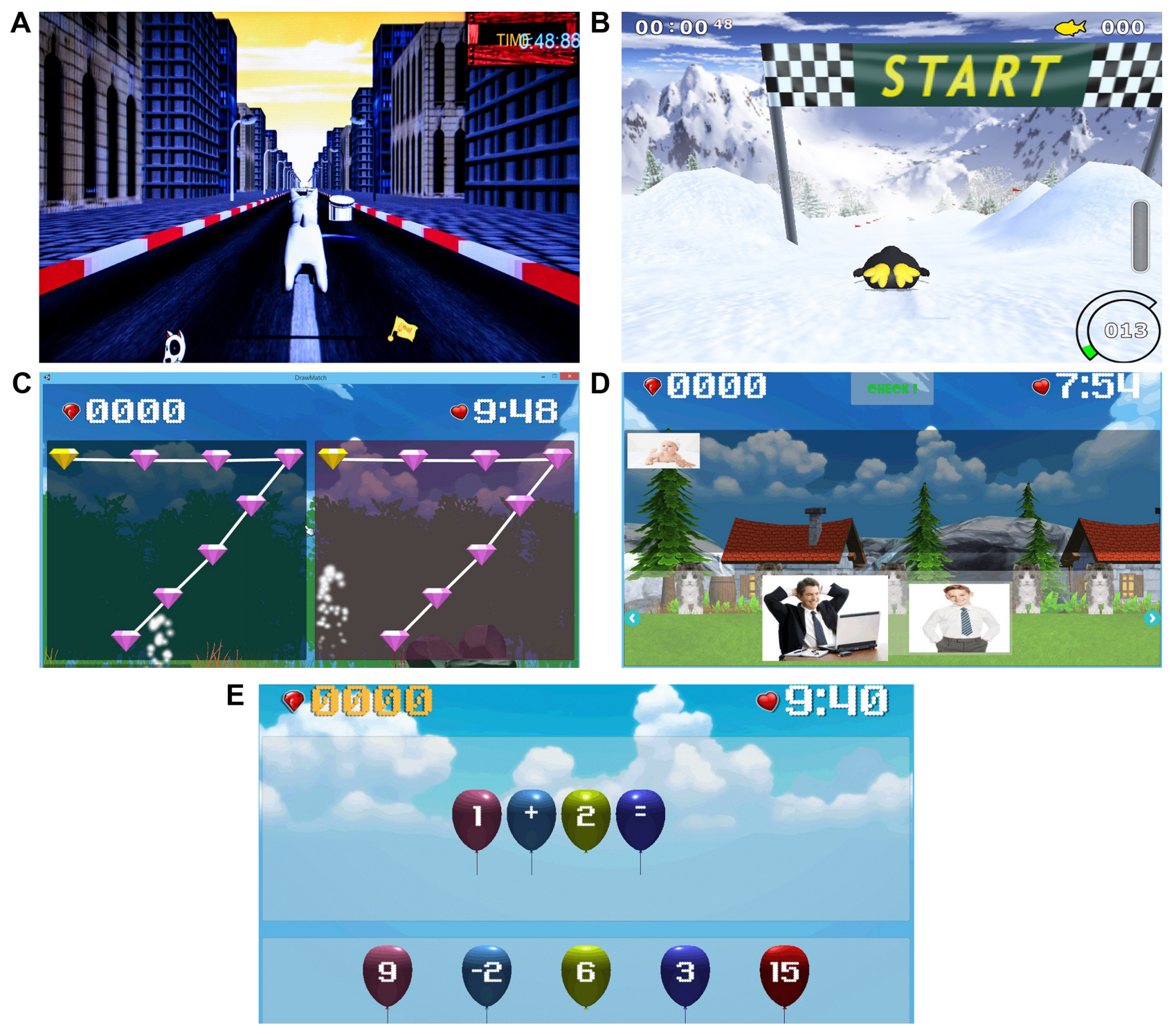

Figure 5 Exergame games.

Note: (A) Dogsperate, (B) Penguin, (C) Both Hands Draw, (D) Recall Mem, and (E) Math.
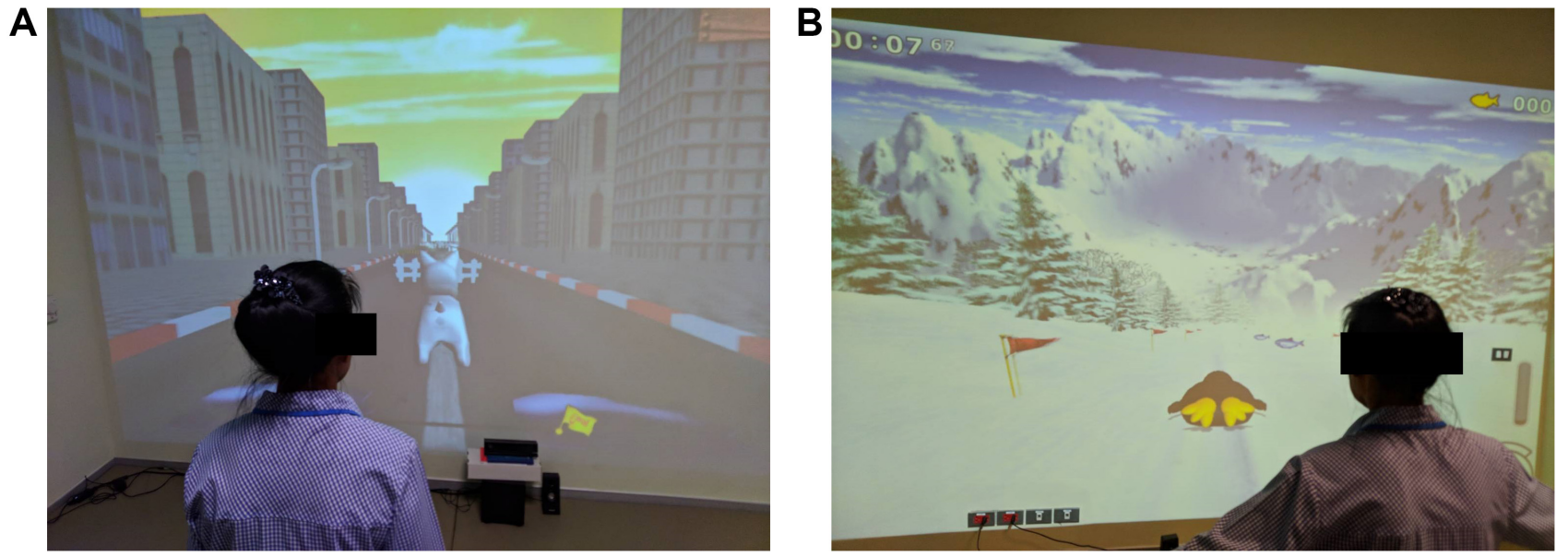

Figure 6 Exergame intervention sessions.

Note: (A) Dogsperate and (B) Penguin. 
the CANTAB tests (pre- and post-intervention) as dependent variables and the three treatment groups, treatment groups $\times$ time, time, healthy elderly controls vs aMCI, education, and age as explanatory variables. The primary outcome variable is the treatment groups $\times$ time effect, and the secondary outcome variables are the effects of time (treatment) and the difference between aMCI and controls. In addition, when the primary GEE analysis was significant, we also computed simple effects to examine the effects of NFT in the separate treatment groups. All results were $p$-corrected for false discovery rate. ${ }^{39}$ The analyses were performed according to the intention-to-treat principle. Tests were two-tailed and a $P$-value of 0.05 was used for statistical significance. All statistical analyses were performed using IBM SPSS windows version 25 . Statistical analyses were conducted in accordance with the International Conference on Harmonisation E9 statistical principles (November 2005).

\section{Results}

\section{Consort flow diagram}

Figure 1 shows the CONSORT flow diagram with the progress of the patients through the trial. Of the 125 participants included in the trial, 6 cases were excluded because they did not comply with inclusion/exclusion criteria. Therefore, 119 patients were entered in the study and were allocated to the CAU ( $n=25,11$ normal controls and 14 aMCI patients), CAU + Exergame ( $n=36,17$ controls and 19 aMCI patients), and CAU + NFT ( $\mathrm{n}=58 ; 26$ controls and 32 aMCI patients) treatment arms. Thus, 54 controls and 65 aMCI patients were enrolled in the trial, while all participants completed the study without any violations of the study protocol. There were no missing data in the CANTAB dataset. Accordingly, we analyzed data of 119 participants treated with CAU only, $\mathrm{CAU}+$ Exergame, and CAU + NFT.

\section{Baseline characteristics of the three treatment groups}

Table 1 compares the demographic, clinical, and CANTAB data between the three study groups. We found no significant differences between these treatment groups in age, education, TMSE, and MOCA scores. There were no significant differences in any of the baseline CANTAB measurements between the three study groups.

Table 2 shows the outcome of the primary GEE analysis with the repeated measurements of the CANTAB tests as dependent variables and the three treatment groups, treatment groups $\times$ time, time, controls vs aMCI, education, and age as explanatory variables. The primary outcome variable is the treatment groups $\times$ time effect, and the secondary outcome variables are the effects of time (treatment) and the difference between aMCI and controls. Table 2 shows the results of these GEE analyses and the $P$-values corrected for false discovery rate. There were no significant effects of time on any of the CANTAB scores.

Table 2 shows that after $p$-correction, there were significant effects of time $\times$ treatment groups on SWM_BER,

Table I Baseline CANTAB measurements in women allocated to three treatment groups

\begin{tabular}{|c|c|c|c|c|c|c|}
\hline Variables & CAU $^{a}$ & CAU + Exergame ${ }^{b}$ & $\mathbf{C A U}+\mathbf{N F T}^{c}$ & $\boldsymbol{F}$ & $d f$ & $P$-value ${ }^{d}$ \\
\hline Age (years) & $70.9(5.1)$ & $73.9(6.2)$ & $71.7(6.5)$ & 1.39 & $2 / 116$ & 0.576 \\
\hline TMSE & $27.9(1.8)$ & $27.5(2.1)$ & $27.3(2.1)$ & 0.69 & $2 / 116$ & 0.732 \\
\hline MOCA & $23.6(0.38)$ & $22.8(4.4)$ & $22.4(4.3)$ & 0.68 & $2 / 116$ & 0.732 \\
\hline Education (years) & II $.4(4.5)$ & $9.4(6.0)$ & $9.0(5.7)$ & 1.73 & $2 / 116$ & 0.576 \\
\hline SWM_BER & $52.8(11.2)$ & $57.6(15.1)$ & $58.3(13.8)$ & 0.74 & $2 / 114$ & 0.732 \\
\hline SWM_STR & $39.0(3.5)$ & $39.6(3.2)$ & $39.3(3.0)$ & 0.09 & $2 / 114$ & 0.916 \\
\hline PRM_COR & $84.8(7.9)$ & $78.5(13.0)$ & $77.5(14.2)$ & 1.54 & $2 / 114$ & 0.576 \\
\hline DMS_COR & $78.1(9.0)$ & $75.7(13.2)$ & $75.9(12.1)$ & 0.11 & $2 / 114$ & 0.916 \\
\hline DMS_MdCOR & $3,684(823)$ & $4,131(1,199)$ & $4,278(1,430)$ & 1.96 & $2 / 114$ & 0.576 \\
\hline DMS_PER & $0.193(0.147)$ & $0.204(0.186)$ & $0.219(0.178)$ & 0.41 & $2 / 114$ & 0.888 \\
\hline RVP_A' & $0.856(0.6 \mathrm{II})$ & $0.82 I(0.695)$ & $0.837(0.055)$ & 1.54 & $2 / 114$ & 0.576 \\
\hline RVP_MdL & $507.6(137.1)$ & $5522(142.1)$ & $5,38.3(157.5)$ & 0.17 & $2 / 144$ & 0.916 \\
\hline SSP_SPAN & $5.16(0.62)$ & $4.44(0.94)$ & $4.88(1.06)$ & 3.16 & $2 / 144$ & 0.576 \\
\hline
\end{tabular}

Notes: ${ }^{\mathrm{a}} \mathrm{No}$ intervention group (only CAU). ${ }^{\mathrm{b}} \mathrm{CAU}+$ Exergame training intervention group. ${ }^{\mathrm{C}} \mathrm{CAU}+$ Neurofeedback cognitive training intervention group. ${ }^{\mathrm{d}}$-value corrected for false discovery rate.

Abbreviations: CANTAB, Cambridge Neuropsychological Test Automated Battery; CAU, care as usual; DMS_COR, Delayed Matching to Sample Total Correct; DMS_MdCOR, Delayed Matching to Sample Median Correct Latency (ms); DMS_PER, Matching to Sample Percent Correct; MOCA, Montreal Cognitive Assessment; NFT, neurofeedback training; PRM_COR, Pattern Recognition Memory Number Correct; RVP_A', Rapid Visual Information Processing A prime; RVP_MdL, Rapid Visual Information Processing Median Correct Latency (ms); SSP_SPAN, Spatial Span Length; SWM_BER, Spatial Working Memory Between Error; SWM_STR, Spatial Working Memory Strategy; TMSE, Thai Mental State Examination. 
Table 2 Results of generalized estimating equation analysis, repeated measurements, with the CANTAB tests scores as dependent variables

\begin{tabular}{|c|c|c|c|c|c|c|c|c|c|}
\hline \multirow[t]{2}{*}{ Variables } & \multicolumn{3}{|c|}{ Time } & \multicolumn{3}{|c|}{ Time $\times$ treatment } & \multicolumn{3}{|c|}{$\mathrm{HC}$ vs aMCl } \\
\hline & $w$ & $d f$ & $P$-value ${ }^{a}$ & $w$ & $d f$ & $P$-value ${ }^{a}$ & $w$ & $d f$ & $P$-value \\
\hline SWM_BER & 0.12 & 1 & 0.963 & 18.68 & 2 & 0.002 & 12.27 & I & 0.002 \\
\hline SWM_STR & 0.34 & 1 & 0.963 & 15.16 & 2 & 0.002 & 11.02 & I & 0.002 \\
\hline PRM_COR & 1.39 & 1 & 0.717 & 1.12 & 2 & 0.642 & $|5.6|$ & 1 & 0.002 \\
\hline DMS_COR & 0.06 & 1 & 0.963 & 1.15 & 2 & 0.642 & 29.57 & 1 & 0.002 \\
\hline DMS_MdCOR & 4.14 & 1 & 0.189 & 1.99 & 2 & 0.642 & 3.25 & 1 & 0.093 \\
\hline DMS_PER & 0.03 & 1 & 0.963 & 0.04 & 2 & 0.979 & 10.75 & 1 & 0.004 \\
\hline RVP_A' & 4.85 & 1 & 0.189 & 31.66 & 2 & 0.002 & 2.34 & 1 & 0.142 \\
\hline RVP_MdL & 0.00 & 1 & 0.989 & 1.46 & 2 & 0.642 & 2.07 & I & 0.150 \\
\hline SSP_SPAN & 0.56 & 1 & 0.963 & 16.03 & 2 & 0.002 & 3.28 & 1 & 0.093 \\
\hline
\end{tabular}

Notes: This table examines the effects of CAU + neurofeedback vs CAU + exergame vs CAU alone (No active intervention). ${ }^{a} A f t e r ~ P$-value correction for false discovery rate.

Abbreviations: aMCl, amnestic mild cognitive impairment; CANTAB, Cambridge Neuropsychological Test Automated Battery; CAU, care as usual; DMS_COR, Delayed Matching to Sample Total Correct; DMS_MdCOR, Delayed Matching to Sample Median Correct Latency (ms); DMS_PER, Matching to Sample Percent Correct; HC, healthy controls; PRM_COR, Pattern Recognition Memory Number Correct; RVP_A', Rapid Visual Information Processing A prime; RVP_MdL, Rapid Visual Information Processing Median Correct Latency (ms); SSP_SPAN, Spatial Span Length; SWM_BER, Spatial Working Memory Between Error; SWM_STR, Spatial Working Memory Strategy; Time, differences in pretreatment vs posttreatment CANTAB scores; Time $\times$ Treatment, interaction time $\times$ three treatment groups.

SWM_STR, RVP_A', and SSP_SPAN. We consequently used analyses of simple effects to examine the effects of time (treatment) on the CANTAB tests in each of the three treatment modalities separately. There were significant effects of treatment in the NFT treatment group on SWM_BER $(F=6.53, d f=1 / 107.2, P=0.012)$, SWM_STR $(F=8.53$, $d f=1 / 105.0, P=0.004)$, and RVP_A $(F=45.02, d f=1 / 109.2$, $P<0.001)$. Figure 7 shows the effects of NFT in the CAU + NFT group only, ie, NFT decreased SWM_BER and SWM STR and increased RVP_A'. There were no significant effects of treatment on these CANTAB test scores in the CAU only and CAU + Exergame treatment groups. There were no significant interactions between diagnosis (aMCI vs controls) and time for any of the CANTAB tests.

There were also significant positive effects of treatment in the CAU + Exergame group on SSP_SPAN ( $F=6.26$, $d f=1 / 65.6, P=0.015$ ), while in the NFT group no significant effects could be detected $(F=3.73, d f=1 / 110, P=0.091)$.

Table 3 shows the results of similar GEE analyses which examined the effects of time $\times$ treatment groups, namely $\mathrm{CAU}+\mathrm{NFT}$ vs CAU + Exergame and CAU alone, while all other explanatory variables were the same as those in Table 2; $P$-values were corrected for false discovery rate. Table 3 shows that there were significant effects of NFT on SWM_BER, SWM_STR, and RVP_A', while CAU + exergame had a significant effect on SSP_SPAN. There were also significant differences in SWM_STR and RVP_A' between participants treated with NFT and the combined CAU + Exergame group and CAU groups.

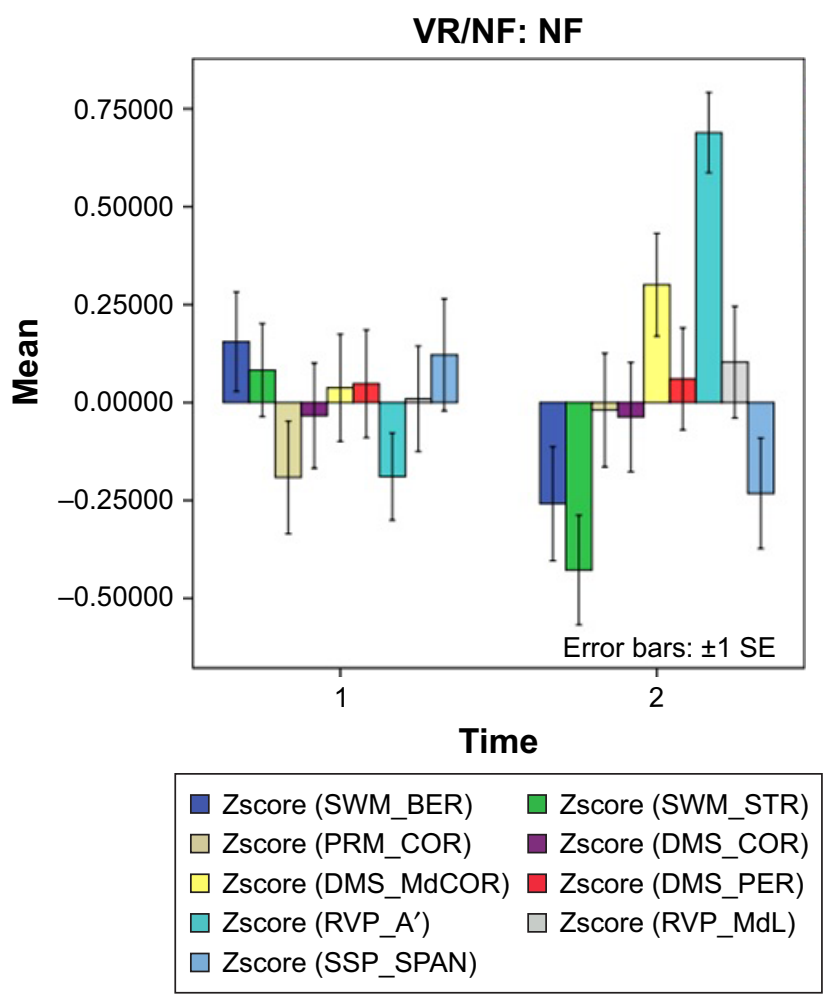

Figure 7 The effects of neurofeedback treatment.

Notes: Shown are the results of the CANTAB tests both before (I) and after (2) treatment. Results are shown as mean (SE) values of the $z$ transformations of the CANTAB test scores.

Abbreviations: CANTAB, Cambridge Neuropsychological Test Automated Battery; DMS_COR, Delayed Matching to Sample Total Correct; DMS_MdCOR, Delayed Matching to Sample Median Correct Latency (ms); DMS_PER, Matching to Sample Percent Correct; PRM_COR, Pattern Recognition Memory Number Correct; RVP_A', Rapid Visual Information Processing A prime; RVP_MdL, Rapid Visual Information Processing Median Correct Latency (ms); SE, standard error; SSP_SPAN, Spatial Span Length; SWM_BER, Spatial Working Memory Between Error; SWM_STR, Spatial Working Memory Strategy. 
Table 3 Results of generalized estimating equation analysis, repeated measurements, with the CANTAB tests as dependent variables

\begin{tabular}{|l|l|l|l|l|l|l|}
\hline \multirow{2}{*}{$\begin{array}{l}\text { CANTAB } \\
\text { tests }\end{array}$} & \multicolumn{3}{|l|}{ Time $\times$ treatment } & \multicolumn{3}{l|}{ NFT vs no-NFT } \\
\cline { 2 - 7 } & $\boldsymbol{W}$ & df & $\boldsymbol{P}$-value & W & df & $\boldsymbol{P}_{\text {-value }}$ \\
\hline SWM_BER & 15.56 & $\mathrm{I}$ & 0.002 & $0.6 \mathrm{I}$ & $\mathrm{I}$ & 0.435 \\
\hline SWM_STR & 9.87 & $\mathrm{I}$ & 0.003 & 5.59 & $\mathrm{I}$ & 0.036 \\
\hline RVP_A' & 28.17 & $\mathrm{I}$ & 0.002 & 18.08 & $\mathrm{I}$ & 0.004 \\
\hline SSP_SPAN & 6.01 & $\mathrm{I}$ & 0.014 & 0.94 & $\mathrm{I}$ & 0.435 \\
\hline
\end{tabular}

Notes: This table examines the effects of CAU + NFT vs the other two treatments, namely CAU + exergame and CAU alone (no-NFT). ${ }^{a}$ After $P$ correction for false discovery rate.

Abbreviations: CANTAB, Cambridge Neuropsychological Test Automated Battery; CAU, care as usual; NFT, neurofeedback training; NFT vs No-NFT, differences in cognitive tests scores between subjects treated with NFT versus those not treated with NFT; RVP_A', Rapid Visual Information Processing A prime; SSP SPAN, Spatial Span Length; SWM_BER, Spatial Working Memory Between Error; SWM_STR, Spatial Working Memory Strategy; Time, differences in pretreatment vs posttreatment CANTAB scores.

\section{Differences in CANTAB test scores between aMCl patients and healthy controls}

Table 2 shows the outcome of the statistical analyses comparing the CANTAB test results in participants with aMCI vs healthy elderly people ( $P$-values were corrected for false discovery rate). Figure 8 shows the mean CANTAB test results (in $z$ transformations) in both aMCI patients and controls. SWM_BER, SWM_STR, and DMS_PER were significantly higher in aMCI than in controls, while PRM_COR and DMS_COR were significantly lower in aMCI.

\section{Discussion}

The major finding of this study is that NFT significantly decreased SWM_BER and SWM_STR tests scores and increased RVP_A' scores. This shows that NFT, but not exergame treatment or CAU, improves working memory, the ability to retain spatial information, and strategy for completing a task, as well as visual sustained attention capability. Importantly, there were no significant differences in the effects of NFT on cognitive functions between healthy elderly and aMCI subjects; both groups improved their working memory, strategy, and sustained attention during NFT treatment. Our results in normal volunteers are in agreement with a review published by Gruzelier showing that NFT may improve cognitive functioning in healthy elderly participants, including memory, executive functions, and sustained attention. ${ }^{19}$ Moreover, the current study shows that NFT resulted in the same effects in participants with aMCI, indicating that these subjects may also benefit from NFT.
Previously, it was shown that some of the NFT effects in MCI on memory performance may be associated with peak alpha frequencies. ${ }^{22}$ All in all, the results show that it is possible to train healthy elderly and aMCI individuals with NFT to enhance specific domains of their cognitive performance.

Most previous research examining the effects of NFT training on CANTAB test results focused on cognitive training in children, especially those with ADHD. ${ }^{40-42}$ The results of these studies indicate improvements mainly in SWM and RVP areas, which is in agreement with our findings in healthy elderly subjects and aMCI patients. Karbach and Verhaeghen reported that NFT may improve working memory executive control in elderly participants. ${ }^{43}$ Enriquez-Geppert et al found that neurocognitive modulation (including NFT) of brain regions, which are crucially involved in specific executive functions, can lead to behavioral benefits in response inhibition, task switching, and memory updating. ${ }^{44}$

The second major finding of this study is that women with aMCI showed disorders in SWM, including strategy, PRM, and short-term visual memory when compared with age-matched healthy elderly women. This indicates that

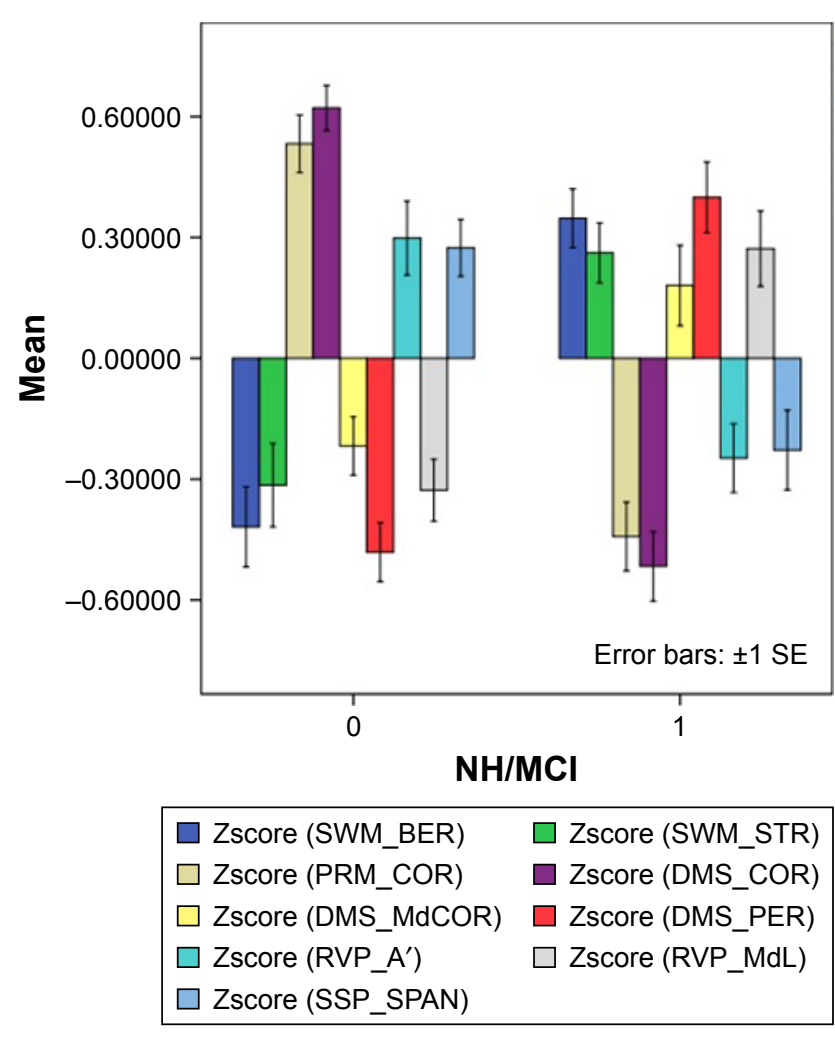

Figure 8 CANTAB test results in both healthy elderly subjects $(0)$ and patients with aMCl (1).

Note: Results are shown as mean (SE) values of the $z$ transformations of the CANTAB test scores.

Abbreviations: aMCl, amnestic mild cognitive impairment; CANTAB, Cambridge Neuropsychological Test Automated Battery; SE, standard error. 
NFT improved some, but not all, features of aMCI such as impairments in PRM and short-term visual memory. On the other hand, NFT significantly improved impaired SWM_STR thereby normalizing the scores to values observed in healthy elderly women. Phrased differently, NFT may be used clinically in aMCI to normalize strategy and improve SWM and sustained attention, which may result in beneficial effects on overall cognitive functions. Therefore, deficits in strategy may be a new indication to treat aMCI patients with NFT.

It is interesting to note that NFT did not have any significant effect on the SSP CANTAB test, which probes the subject's attention span and ability to assess working memory, while exergame treatment, but not CAU, significantly improved the SSP test outcome. This suggests that exergame significantly improves attention span and the ability to assess working memory in both healthy elderly and aMCI patients. Previously, it was shown that exergame may improve cognitive functioning, in particular, executive control skills. ${ }^{45,46}$ These beneficial effects may be explained by recent knowledge that aerobic fitness improves cognitive performance via increased cerebral circulation, increased neurotransmitter availability, and enhanced physiological and neurological processes. ${ }^{47}$ Exergames may also improve cognitive control of attention, which in turn could improve cognitive functioning. ${ }^{48}$ Other cognitive functions that could benefit from exergaming include visual-spatial skills including spatial relations, visualization, perceptual speed, and 3D rotation skills. ${ }^{49}$

All in all, our exergame intervention approach potentially improved speedy reaction and decision-making capability of both aMCI and healthy elderly women. Therefore, it may be speculated that a combined treatment of NFT + exergame may result in a better response than each treatment alone. In this regard, it is interesting to note that some researchers examined the combined effects of cognitive and physical training. ${ }^{50-52}$

The results of the present study should be interpreted with reference to its limitations and strengths. Firstly, the main purpose of the current study was to examine a new game-based NFT system for cognitive enhancement with a consumer-grade EEG acquisition device, called "Emotiv EPOC", one of the low-cost EEG headsets available on the market. However, there are some clinicians and researchers in the field who are concerned about the quality of consumergrade EEG devices for clinical use. In this respect, several studies compared the EEG signal quality of Emotiv EPOC with that of medical- and research-grade devices. Badcock et al showed that the morphology of ERP signals of Emotiv EPOC devices was very similar to that of the signal acquired from a research-grade EEG system (Neuscan). ${ }^{53}$ There were only few differences of peak amplitude and latency estimates between the two systems. Duvinage et al found that the EEG signal quality of Emotiv EPOC is somewhat less than that of a medical-grade system (ANT Neuro System), although the results show that the Emotiv headset can be used in noncritical applications such as games for rehabilitations. ${ }^{54}$ Secondly, our interventional study was performed on women only, and therefore a follow-up study should be performed to examine the effects of NFT in elderly healthy males and males with aMCI. Thirdly, it would have been even more interesting if we had used more cognitive tests, for example, using the Consortium to Establish a Registry for AD tests to probe semantic and episodic memory, and word recall and recognition. ${ }^{55}$ The strengths of this study are that we examined the efficacy of NFT in a large study sample and compared NFT with CAU and exergame and adjusted for possible effects of age and education.

\section{Conclusions}

In conclusion, our experimental results show that NFT intervention has a clinical efficacy in improving selected domains of cognitive functions in healthy elderly women and women with aMCI, namely SWM, including strategy, and sustained attention capability. Our study shows that our game-based NFT system using an inexpensive consumer-grade EEG headset can be implemented by aMCI patients and healthy elderly people at home to train strategy, working memory, and sustained attention. Consequently, the proposed gamebased NFT system is potentially an alternative treatment for enhancing cognitive performance in aMCI and healthy elderly subjects. Interestingly, this study also showed that exergame, but not NFT, significantly improved attention span and the ability to assess working memory. It should be examined whether the combination of NFT + exergame may have an augmenting effect on cognitive performance above and beyond the effects of each treatment alone.

\section{Acknowledgment}

The authors express their gratitude to the 125 volunteers who participated in this NFT study program.

\section{Author contributions}

All authors contributed toward data analysis, drafting and critically revising the paper, gave final approval of the version to be published and agree to be accountable for all aspects of the work.

\section{Disclosure}

The authors report no conflicts of interests in this work. 


\section{References}

1. Fabiani GE, Mcfarland DJ, Wolpaw JR, Pfurtscheller G. Conversion of EEG activity into cursor movement by a brain-computer interface (BCI). IEEE Trans Neural Syst Rehabil Eng. 2004;12(3):331-338.

2. Prasad G, Herman P, Coyle D, Mcdonough S, Crosbie J. Using motor imagery based brain-computer interface for post-stroke rehabilitation. 4th International IEEE/EMBS Conference on Neural Engineering. 2009:258-262.

3. Montesano L, Díaz M, Bhaskar S, Minguez J. Towards an intelligent wheelchair system for users with cerebral palsy. IEEE Trans Neural Syst Rehabil Eng. 2010;18(2):193-202.

4. Wang W, Collinger JL, Perez MA, et al. Neural interface technology for rehabilitation: exploiting and promoting neuroplasticity. Phys Med Rehabil Clin N Am. 2010;21(1):157-178.

5. de Ruiter MA, Schouten-van Meeteren AY, van Mourik R, et al. Neurofeedback to improve neurocognitive functioning of children treated for a brain tumor: design of a randomized controlled double-blind trial. BMC Cancer. 2012;12(1):581.

6. Gehring K, Roukema JA, Sitskoorn MM. Review of recent studies on interventions for cognitive deficits in patients with cancer. Expert Rev Anticancer Ther. 2012;12(2):255-269.

7. Faucounau V, Wu YH, Boulay M, de Rotrou J, Rigaud AS. Cognitive intervention programmes on patients affected by mild cognitive impairment: a promising intervention tool for MCI? J Nutr Health Aging. 2010;14(1):31-35

8. Chen YL, Chen C-L, Chang WH, Wong M-K, Tung F-T, Kuo TS. The development of a biofeedback training system for cognitive rehabilitation in cerebral palsy. Proceedings of the 19th IEEE Annual International Conference on Engineering in Medicine and Biology. 1997;5:1919-1920.

9. Budzynski HT. Introduction to Quantitative EEG and Neurofeedback: Advanced Theory and Applications. 2nd ed. New York: Elsevier; 2009

10. Duric NS, Assmus J, Gundersen D, Elgen IB. Neurofeedback for the treatment of children and adolescents with ADHD: a randomized and controlled clinical trial using parental reports. BMC Psychiatry. 2012;12(1):107.

11. Gevensleben H, Holl B, Albrecht B, et al. Neurofeedback training in children with ADHD: 6-month follow-up of a randomised controlled trial. Eur Child Adolesc Psychiatry. 2010;19(9):715-724.

12. Arns M, de Ridder S, Strehl U, Breteler M, Coenen A. Efficacy of neurofeedback treatment in ADHD: the effects on inattention, impulsivity and hyperactivity: a meta-analysis. Clin EEG Neurosci. 2009; 40(3):180-189.

13. Tang YY, Posner MI. Attention training and attention state training. Trends Cogn Sci. 2009;13(5):222-227.

14. Pondé MP, Cruz-Freire AC, Silveira AA. Relationship between learning problems and attention deficit in childhood. J Atten Disord. 2012; 16(6):505-509.

15. Becerra J, Fernández T, Roca-Stappung M, et al. Neurofeedback in healthy elderly human subjects with electroencephalographic risk for cognitive disorder. J Alzheimers Dis. 2012;28(2):357-367.

16. Zoefel B, Huster RJ, Herrmann CS. Neurofeedback training of the upper alpha frequency band in EEG improves cognitive performance. Neuroimage. 2011;54(2):1427-1431.

17. Haddadi P, Rostami R, Moradi A, Pouladi F. Neurofeedback training to enhance learning and memory in patients with cognitive impairment. Procedia Soc Behav Sci. 2011;30:608-610.

18. Engelbregt HJ, Keeser D, van Eijk L, et al. Short and long-term effects of sham-controlled prefrontal EEG-neurofeedback training in healthy subjects. Clin Neurophysiol. 2016;127(4):1931-1937.

19. Gruzelier JH. EEG-neurofeedback for optimising performance. I: A review of cognitive and affective outcome in healthy participants. Neurosci Biobehav Rev. 2014;44:124-141.

20. Luijmes RE, Pouwels S, Boonman J. The effectiveness of neurofeedback on cognitive functioning in patients with Alzheimer's disease: preliminary results. Neurophysiol Clin. 2016;46(3):179-187.
21. Hohenfeld C, Nellessen N, Dogan I, et al. Cognitive improvement and brain changes after real-time functional MRI neurofeedback training in healthy elderly and prodromal Alzheimer's disease. Front Neurol. 2017;9(8):384.

22. Lavy Y, Dwolatzky T, Kaplan Z, Guez J, Todder D. Neurofeedback improves memory and peak alpha frequency in individuals with mild cognitive impairment. Appl Psychophysiol Biofeedback. Epub 2018 Oct 4.

23. Anderson-Hanley C, Barcelos NM, Zimmerman EA, et al. The aerobic and cognitive exercise study (ACES) for community-dwelling older adults with or at-risk for mild cognitive impairment (MCI): neuropsychological, neurobiological and neuroimaging outcomes of a randomized clinical trial. Front Aging Neurosci. 2018;10(10):76.

24. Morris JC. The clinical dementia Rating (CDR). Neurology. 1993;43(11): 2412-2414.

25. Petersen RC. Mild cognitive impairment as a diagnostic entity. J Intern Med. 2004;256(3):183-194.

26. Mckhann G, Drachman D, Folstein M, Katzman R, Price D, Stadlan EM. Clinical diagnosis of Alzheimer's disease: report of the NINCDSADRDA work group under the auspices of department of health and Human Services Task Force on Alzheimer's disease. Neurology. 1984;34(7):939-944.

27. American Psychiatric Association. Diagnostic and statistical manual of mental disorders (DSM-5). Available from: https://www.psychiatry. org/psychiatrists/practice/dsm. Accessed December 5, 2018.

28. Train the Brain Forum Committee. Thai Mental State Examination (TMSE). Siriraj Hosp Gaz. 1993;45:359-374.

29. The Montreal Cognitive Assessment (MoCA). Available from: http:// www.mocatest.org. Accessed December 5, 2018.

30. Cambridge Neuropsychological Test Automated Battery (CANTAB). Available from: http://www.cantab.com. Accessed December 5, 2018.

31. Emotiv EPOC Headset [Online]; 2015. Available from: https://www. emotiv.com/files/Emotiv-EPOC-Product-Sheet-2014.pdf. Accessed January 30, 2019.

32. Hagemann D, Naumann E. The effects of ocular artifacts on (lateralized) broadband power in the EEG. Clin Neurophysiol. 2001;112(2): 215-231.

33. Siemiomow V, Shagal V, Yue GH. Single-Trial EEG-EMG coherence analysis reveals muscle fatigue-related progressive alterations in corticomuscular coupling. IEEE Trans Neural Syst Rehabil Eng. 2010; 18:97-106.

34. Goncharova II, Mcfarland DJ, Vaughan TM, Wolpaw JR. EMG contamination of EEG: spectral and topographical characteristics. Clin Neurophysiol. 2003;114(9):1580-1593.

35. Jirayucharoensak S, Israsena P, Pan-Ngum S. Online EEG artifact suppression for neurofeedback training systems. Biomedical Engineering International Conference. 2013:1-5.

36. Yaomanee K, Pan-ngum S, Ayuthaya PI. Brain signal detection methodology for attention training using minimal EEG channels. InICT and Knowledge Engineering (ICT \& Knowledge Engineering), 2012 10th International Conference on 2012 Nov 21 (pp. 84-89). IEEE.

37. Vázquez Marrufo M, Vaquero E, Cardoso MJ, Gómez CM. Temporal evolution of $\alpha$ and $\beta$ bands during visual spatial attention. Cogn Brain Res. 2001;12(2):315-320.

38. Klimesch W. EEG alpha and theta oscillations reflect cognitive and memory performance: a review and analysis. Brain Res Rev. 1999;29(2-3): 169-195.

39. Benjamini Y, Hochberg Y. Controlling the false discovery rate: a practical and powerful approach to multiple testing. J R Stat Soc Series B. 1995; 57(1):289-300.

40. Ensafi E, Rostami R, Dolatshahi B, Poursharifi H, Nouri M. The effectiveness of neurofeedback on the working memory in children with ADHD. Pract Clin Psychol. 2014;2(3):128-134.

41. Shiva T, Hasan S. Rapid Visual Processing and Spatial Working Memory improvement after QEEG-based Neurofeedback Training in ADHD Children: Evidence from CANTAB. In 6th International Congress on Child and Adolescent Psychiatry 2013 Sep 17. Tabtiz university of medical sciences. 
42. Israsena P, Hemrungrojn S, Sukwattanasinit N, Maes M. Development and evaluation of an interactive electro-encephalogram-based neurofeedback system for training attention and attention defects in children. $J$ Med Imaging Health Inform. 2015;5(5):1045-1052.

43. Karbach J, Verhaeghen P. Making working memory work: a metaanalysis of executive-control and working memory training in older adults. Psychol Sci. 2014;25(11):2027-2037.

44. Enriquez-Geppert S, Huster RJ, Herrmann CS. Boosting brain functions: improving executive functions with behavioral training, neurostimulation, and neurofeedback. Int J Psychophysiol. 2013;88(1):1-16.

45. Hertzog C, Kramer AF, Wilson RS, Lindenberger U. Fit body, fit mind? Sci Am Mind. 2009;20(4):24-31.

46. Staiano AE, Abraham A, Calvert SL. Improved executive functioning from Wii Active exergame play: A study and results. In Annual Games for Health Conference, Boston, MA 2010.

47. Etnier JL, Nowell PM, Landers DM, Sibley BA. A meta-regression to examine the relationship between aerobic fitness and cognitive performance. Brain Res Rev. 2006;52(1):119-130.

48. Hillman CH, Pontifex MB, Raine LB, Castelli DM, Hall EE, Kramer AF. The effect of acute treadmill walking on cognitive control and academic achievement in preadolescent children. Neuroscience. 2009; 159(3):1044-1054.

49. Subrahmanyam K, Greenfield PM. Effect of video game practice on spatial skills in girls and boys. J Appl Dev Psychol. 1994;15(1):13-32.
50. Maillot P, Perrot A, Hartley A. Effects of interactive physical-activity video-game training on physical and cognitive function in older adults. Psychol Aging. 2012;27(3):589-600.

51. Kayama H, Okamoto K, Nishiguchi S, Yamada M, Kuroda T, Aoyama T. Effect of a kinect-based exercise game on improving executive cognitive performance in community-dwelling elderly: case control study. J Med Int Res. 2014;16(2):e61.

52. Bamidis PD, Fissler P, Papageorgiou SG. Gains in cognition through combined cognitive and physical training: the role of training dosage and severity of neurocognitive disorder. Front Aging Neurosci. 2012;7:152.

53. Badcock NA, Mousikou P, Mahajan Y, de Lissa P, Thie J, Mcarthur G. Validation of the Emotiv EPOC ${ }^{\circledR}$ EEG gaming system for measuring research quality auditory ERPs. Peer J. 2013;19:e38.

54. Duvinage M, Castermans T, Dutoit T, et al. A P300-based quantitative comparison between the Emotiv EPOC Headset and a medical EEG device. BioMed Eng Online. 2012;765.

55. Tunvirachaisakul C, Supasitthumrong T, Tangwongchai S, et al. Characteristics of mild cognitive impairment using the Thai version of the consortium to establish a registry for Alzheimer's disease tests: a multivariate and machine learning study. Dement Geriatr Cogn Disord. 2018;45(1-2):38-48.
Clinical Interventions in Aging

\section{Publish your work in this journal}

Clinical Interventions in Aging is an international, peer-reviewed journal focusing on evidence-based reports on the value or lack thereof of treatments intended to prevent or delay the onset of maladaptive correlates of aging in human beings. This journal is indexed on PubMed Central, MedLine,

\section{Dovepress}

CAS, Scopus and the Elsevier Bibliographic databases. The manuscript management system is completely online and includes a very quick and fair peer-review system, which is all easy to use. Visit http://www.dovepress. com/testimonials.php to read real quotes from published authors. 